\title{
Lentivirally overexpressed T-bet regulates T-helper cell lineage commitment in chronic hepatitis B patients
}

\author{
XUENI LIU, ZHENGHAO TANG, YI ZHANG, JIANJUN HU, DAN LI, GUOQING ZANG and YONGSHENG YU \\ Department of Infectious Diseases, Sixth People's Hospital, Shanghai Jiaotong University, Shanghai 200233, P.R. China
}

Received January 12, 2012; Accepted May 2, 2012

DOI: $10.3892 / \mathrm{mmr} .2012 .905$

\begin{abstract}
Chronic hepatitis B virus (HBV) infection is commonly considered to occur as a result of disturbance of the immune system. T-box expressed in T cells (T-bet) is an essential transcription factor for $\mathrm{T}$ helper (Th) cell differentiation and function. The aim of this study was to investigate the effect of T-bet overexpression on Th cell differentiation and the possible mechanism in chronic hepatitis $\mathrm{B}(\mathrm{CHB})$ patients. $\mathrm{CD}^{+} \mathrm{T}$ cells from the peripheral blood of $23 \mathrm{CHB}$ patients, 8 acute hepatitis $\mathrm{B}$ (AHB) patients and 10 healthy controls were isolated. T-bet mRNA expression of $\mathrm{CD}^{+} \mathrm{T}$ cells was detected by quantitative real-time polymerase chain reaction (PCR). The T-bet DNA fragment was subcloned into the pGC-FU vector containing GFP to generate a recombinant lentiviral vector, pGC-FU-T-bet, while a no-load pGC-FU vector was used as the negative control. After transduction into $\mathrm{CD} 4^{+} \mathrm{T}$ cells from another 22 CHB patients, the induction of Th1- and Th2-type cytokines was assayed by an enzyme-linked immunosorbent assay (ELISA), and RT-PCR and western blot analysis were used to measure the mRNA and transcription levels of H2.0like homeobox (HLX1), GATA-3 and STAT-6. T-bet mRNA expression in $\mathrm{CD}^{+} \mathrm{T}$ cells from $\mathrm{AHB}$ patients was enhanced compared with CHB patients and healthy controls. Th1-type cytokines and HLX1 expression was upregulated, while Th2-type cytokines and GATA-3 and STAT-6 expression was repressed after lentiviral introduction of T-bet. In conclusion, lentivirally overexpressed T-bet regulates Th cell lineage commitment in CHB patients, which may be mediated by regulating HLX1, GATA-3 and STAT-6 expression.
\end{abstract}

\section{Introduction}

Viral hepatitis is a necroinflammatory liver disease of variable severity. Persistent infection of hepatitis B virus (HBV)

Correspondence to: Dr Yongsheng Yu or Dr Guoqing Zang, Department of Infectious Diseases, Sixth People's Hospital, Shanghai Jiaotong University, Shanghai 200233, P.R. China

E-mail: yuyongsheng@medmail.com.cn

E-mail: zangguoqin@126.com

Key words: chronic hepatitis B, T-bet, T helper cells, H2.0-like homeobox, GATA-3, STAT-6 is often associated with chronic liver disease that leads to the development of cirrhosis and hepatocellular carcinoma. The persistence of $\mathrm{HBV}$ infection is commonly considered as a result of an inadequate host immune response. It is generally acknowledged that the cellular immune response contributes to viral clearance, particularly T-cell immunity for HBV (1). The correlation between viral spread and $\mathrm{CD} 4^{+} \mathrm{T}$ cell priming determines the outcome of $\mathrm{HBV}$ infection (2). $\mathrm{CD}^{+}{ }^{+} \mathrm{T}$ cells are classified into two types of $\mathrm{T}$ helper cells on the activation of a certain antigen: Th1 and Th2. These cells differ in their pattern of secreted cytokines. Th1 cells secrete interferon- $\gamma($ IFN- $\gamma)$, interleukin-2 (IL-2) and tumor necrosis factor- $\beta$ (TNF- $\beta$ ), which aid in the clearance of intracellular pathogens, while Th2 cells secrete IL-4, IL-5 and IL-10 which help alleviate extracellular infections.

Some discoveries have led to the identification of two major transcription factors, T-bet and GATA-3, as the master regulators of the Th1 and Th2 differentiation programs, respectively. T-bet is required for differentiation of Th1 cells defined by the expression of signature Th1 genes, including the cytokine IFN- $\gamma$ and the cell surface receptor $(3,4)$. Th2 cell differentiation is usually associated with GATA-3 upregulation, while deletion of the GATA-3 gene blocks Th2 commitment (5). The H2.0-like homeobox (HLX1) is a member of the homeobox gene family which contains a helixturn-helix DNA-combination domain consisting of 61 amino acids, whose expression closely correlates with the immune system. It has synergistic effects on Th1 cell development with T-bet. Activation of the transcription factor STAT- 6 promotes Th2 cell differentiation mediated by inducing expression of GATA-3 and may directly transactivate the IL-4 gene (6).

However, the link between T-bet and the pathogenesis of $\mathrm{CHB}$ remains unclear. In order to demonstrate the role of T-bet in patients with chronic HBV infection, we analyzed the expression of $\mathrm{T}$-bet in $\mathrm{CD} 4^{+} \mathrm{T}$ cells and the effect of overexpression of $\mathrm{T}$-bet on $\mathrm{Th}$ cell differentiation in $\mathrm{CHB}$ patients.

\section{Patients and methods}

Patients. Between December 2010 and June 2011, 45 CHB patients (31 males and 14 females; average age, 38.0 12.1 years; 34 with positive $\mathrm{HBeAg}$, 11 with negative $\mathrm{HBeAg}$; average ALT, $227.1 \pm 163.3 \mathrm{IU} / \mathrm{l})$, and $8 \mathrm{AHB}$ patients ( 6 males and 2 females; average age, $45.0 \pm 14.5$ years; average ALT, 1775.4 \pm 835.7 IU/l) 
admitted to The Sixth People's Hospital Affiliated to Shanghai Jiaotong University, Shanghai, China, and 10 healthy controls were enrolled in the study. Of all subjects, 23 CHB patients, 8 AHB patients and 10 healthy controls were included in the T-bet mRNA expression assay, while $22 \mathrm{CHB}$ patients were included in the lentiviral vector transduction study. All CHB patients were seropositive for $\mathrm{HBsAg}$ and $\mathrm{HBcAb}$ for more

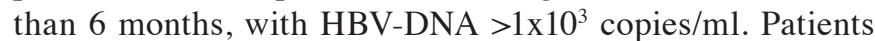
with hepatitis $C$ virus, hepatitis $D$ virus or human immunodeficiency virus (HIV) infection, autoimmune disease, or with a history of receiving antiviral or immunosuppressive therapy within 6 months or alcohol abuse were excluded.

This study was approved by the local ethics committee, and all patients provided written informed consent prior to study enrollment.

Enrichment of $\mathrm{CD}^{+} \mathrm{T}$ cells. A RosetteSep human $\mathrm{CD} 4^{+} \mathrm{T}$ cell enrichment cocktail (StemCell Technologies, Vancouver, BC, Canada) was used for enrichment of $\mathrm{CD} 4^{+} \mathrm{T}$ cells from whole blood. A total of $1 \mathrm{ml}$ RosetteSep cocktail was added to $20 \mathrm{ml}$ peripheral sodium heparin-anticoagulant blood samples, which cross-linked unwanted cells to red blood cells. Whole blood was diluted with an equal volume of phosphate-buffered saline (PBS), layered over $20 \mathrm{ml}$ Ficoll-Paque (StemCell Technologies), and centrifuged for $20 \mathrm{~min}$ at $1200 \mathrm{x} \mathrm{g}$ at room temperature. $\mathrm{CD} 4{ }^{+} \mathrm{T}$ cells were enriched at the sample/medium interface. The purity of the sorted population was detected by flow cytometry and was consistently greater than $80 \%$.

Real time-PCR for T-bet mRNA. Total cellular RNA was extracted using RNAiso reagent (Takara Bio Inc., Otsu, Japan). Using the PrimeScript ${ }^{\circledR}$ RT reagent kit (Perfect Real Time) (Takara Bio Inc.) and following the manufacturer's instructions, reverse transcription was performed using $1 \mu \mathrm{g}$ of total RNA mixed with $5 \mu \mathrm{l}$ of $5 \mathrm{X}$ PrimeScript buffer, $1 \mu 1$ of PrimeScript ${ }^{\circledR}$ RT Enzyme mix I primed with $1 \mu 1$ of Oligo dT and $1 \mu \mathrm{l}$ of Random 6 mers at $37^{\circ} \mathrm{C}$ for $15 \mathrm{~min}$ and $85^{\circ} \mathrm{C}$ for $5 \mathrm{sec}$. A total of $0.5 \mu 1$ of cDNA was used for real time-PCR using SYBR Premix Ex Taq (Takara Bio Inc.) with primers specific for genes encoding T-bet as follows: forward, 5'-GGTTGCGGAGACATGCTGA-3'; reverse, 5'-GTAGGCGTAGGCTCCAAGG-3'. The amplification conditions were as follows: inactivation for $5 \mathrm{~min}$ at $95^{\circ} \mathrm{C}$ followed by 40 cycles of amplification of $10 \mathrm{sec}$ at $95^{\circ} \mathrm{C}$, $20 \mathrm{sec}$ at $60^{\circ} \mathrm{C}$ and $20 \mathrm{sec}$ at $72^{\circ} \mathrm{C}$. T-bet mRNA expression was normalized to the transcript levels of the internal control, the housekeeping gene GAPDH, with primers as follows: forward, 5'-ATGGGGAAGGTGAAGGTCG-3'; reverse, 5'-GGGGTCATTGATGGCAACAATA-3'. The amount of target was calculated by $2^{-\Delta \Delta C t}$. Three parallel reactions of each sample and internal control were run.

Lentiviral vector transduction. Two recombinant lentiviral vectors were constructed; one vector expressing T-bet and GFP (pGC-FU-T-bet) and the other containing GFP only (pGC-FU). There were two groups for infection, pGC-FU-T-bet and pGC-FU. CD4 ${ }^{+} \mathrm{T}$ cells were seeded at a density of $1 \times 10^{6}$ cells/well in a $24-w e l l$ plate, then activated with $25 \mu \mathrm{l}$ Dynabeads $^{\circledR}$ Human T-Activator CD3/CD28 and expanded with $30 \mathrm{U} / \mathrm{ml}$ rIL-2 (PeproTech Inc., Rocky Hill, NJ, USA).
After $24 \mathrm{~h}$ of culture, $\mathrm{CD} 4^{+} \mathrm{T}$ cells were infected with $200 \mu \mathrm{l} /$ well of either lentiviral vector $\left(1 \times 10^{8} / \mathrm{ml}\right)$ with $100 \mu$ l Polybrene (50 $\mu \mathrm{g} / \mathrm{ml}$; Sigma, St. Louis, MO, USA). Infected cells were examined daily until GFP expression was evident and the supernatants were collected for the cytokine assays, while the cells were collected for the HLX1, GATA-3 and STAT-6 mRNA and protein assays every other day.

Enzyme-linked immunosorbent assay (ELISA) for cytokine assay. Cultured supernatants of infected $\mathrm{CD} 4^{+} \mathrm{T}$ cells were collected on days 3, 5 and 7, and ELISA kits (R\&D Systems Inc., Minneapolis, MN, USA) were used to quantify the concentrations of IFN- $\gamma$, IL-2, IL-4 and IL-10.

Real time-PCR for HLX1, GATA-3 and STAT-6 mRNA. HLX1, GATA-3 and STAT- 6 mRNA were assayed by real time-PCR using the method previously described. The sequences of the primers were as follows: HLX1 forward, 5'-GCAGCAATC ACCAACGCAG-3'; reverse, 5'-GGGTCAAATTCCGCA GACAAA-3'; GATA-3 forward, 5'-GTGCTTTTTAACATC GACGGTC-3'; reverse, 5'-AGGGGCTGAGATTCCAGGG-3'; and STAT-6 forward, 5'-GCCAAAGCCCTAGTGCTGAA-3'; reverse, 5'-GACGAGGGTTCTCAGGACTTC-3'.

Western blot analysis for HLX1, GATA-3 and STAT-6. Total proteins were extracted using RIPA Lysis Buffer (Beyotime Institute of Biotechnology, Jiangsu, China), and determined using bicinchoninic acid (BCA) protein assay kits (Beyotime Institute of Biotechnology), loaded onto $10 \%$ SDS-polyacrylamide gels and transferred to a polyvinylidene difluoride membrane (PVDF) via electroblotting. The membranes were incubated with rabbit anti-HLX1 $(1: 1,200$; Abbiotec, San Diego, CA, USA) and horseradish-peroxidase (HRP)-conjugated anti-GAPDH (1:10,000; Abbiotec), respectively, followed by incubation with HRP-conjugated sheep anti-rabbit antibody (1:10,000; Abbiotec). Specific protein bands on the membranes were visualized using the enhanced chemiluminescence method (Amersham, Piscataway, NJ, USA), and analyzed using Image $\mathbf{J}$ software (version 1.6 NIH) to determine the relative levels of HLX1 defined as the optical density ratio of HLX1 over GAPDH. Expression of GATA-3 and STAT- 6 was also detected in this way using rabbit antiGATA-3 (1:3,000; Epitomics, Inc., Burlingame, CA, USA) and rabbit anti-STAT-6 (1:500; Epitomics, Inc.), respectively.

Statistical analysis. All data are expressed as mean \pm standard deviation (SD), and were analyzed with SPSS 13.0 for Windows. Correlations were evaluated using the Pearson's correlation test. Comparisons between groups were performed using the independent-samples t-test or by one-way ANOVA for experiments with more than two subgroups. Two-tailed $\mathrm{P}<0.05$ was considered to indicate a statistically significant difference.

\section{Results}

T-bet mRNA expression was significantly repressed in $C H B$ patients compared with $A H B$ patients. To investigate the correlation between T-bet and chronic HBV infection, T-bet mRNA from CHB patients $(n=23)$, AHB patients $(n=8)$ and healthy controls $(n=10)$ were detected by RT-PCR. The expres- 


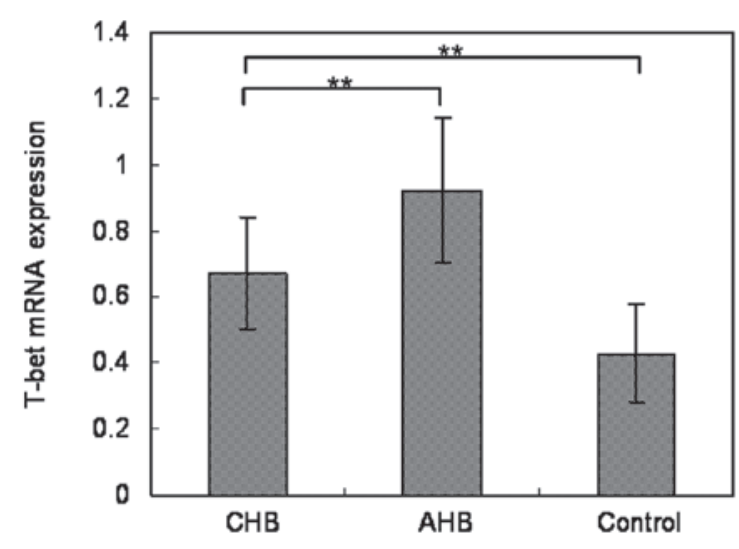

Figure 1. T-bet mRNA expression in $\mathrm{CD} 4^{+} \mathrm{T}$ cells from chronic hepatitis $\mathrm{B}$ (CHB) patients $(n=23)$, acute hepatitis B (AHB) patients $(n=8)$ and healthy controls $(n=10)\left({ }^{* *} \mathrm{P}<0.01\right)$. The results of T-bet mRNA expression demonstrated relative expression of T-bet normalized to GAPDH. T-bet, T-box expressed in T cells.

sion of T-bet mRNA in CD4 ${ }^{+} \mathrm{T}$ cells from the peripheral blood of CHB patients was demonstrated to be lower than that in the AHB patients $(\mathrm{P}<0.01)$, but higher than that in the healthy controls $(\mathrm{P}<0.01)$ (Fig. 1).

Lentivirally overexpressed T-bet regulates Th1/Th2 cytokine expression in $\mathrm{CHB}$ patients. Cultured supernatants of $\mathrm{CD}^{+}$ $\mathrm{T}$ cells on days 3, 5 and 7 after infection were measured by ELISA. Compared with pGC-FU transduction, IFN- $\gamma$ expression following pGC-FU-T-bet transduction was significantly upregulated after 3 days $(\mathrm{P}<0.05), 5$ days $(\mathrm{P}<0.01)$ and 7 days $(\mathrm{P}<0.01)$ (Fig. 2). There was no difference in IL-2 expression at day 3, but expression was significantly enhanced at days 5 $(\mathrm{P}<0.05)$ and $7(\mathrm{P}<0.05)$. Production of IL-4 $(\mathrm{P}<0.05)$ and IL-10 $(\mathrm{P}<0.01)$ was significantly downregulated at day 5 , but was not statistically different at days 3 or 7 . These results demonstrated that the Th1-type cytokines, IFN- $\gamma$ and IL-2, were increased, while Th2-type cytokines, IL-4 and IL-10, were decreased from $\mathrm{CD}^{+}{ }^{+} \mathrm{T}$ cells of $\mathrm{CHB}$ patients after pGC-FU-T-bet transduction. This indicated that lentivirally overexpressed T-bet may regulate Th1/Th2 differentiation and function.

Lentivirally overexpressed T-bet enhances HLX1 expression and represses GATA-3 and STAT-6 expression. To investigate the mechanism of T-bet overexpression regulating Th1/Th2 differentiation, the expression of HLX1, GATA-3 and STAT- 6 mRNA were detected by RT-PCR and the proteins were detected by western blot analysis at day 5 after transfection. The results revealed that expression of HLX1 was significantly upregulated after pGC-FU-T-bet transduction $(\mathrm{P}<0.01)$, while expression of GATA-3 and STAT- 6 were downregulated $(\mathrm{P}<0.01)$ (Figs. 3 and 4). This indicated that HLX1, GATA-3 and STAT-6 may be involved in the process of lentivirally overexpressed T-bet regulating Th1/Th2 differentiation.

\section{Discussion}

Persistent HBV infection is characterized by a weak adaptive immune response, considered to be due to inefficient $\mathrm{CD}^{+}$
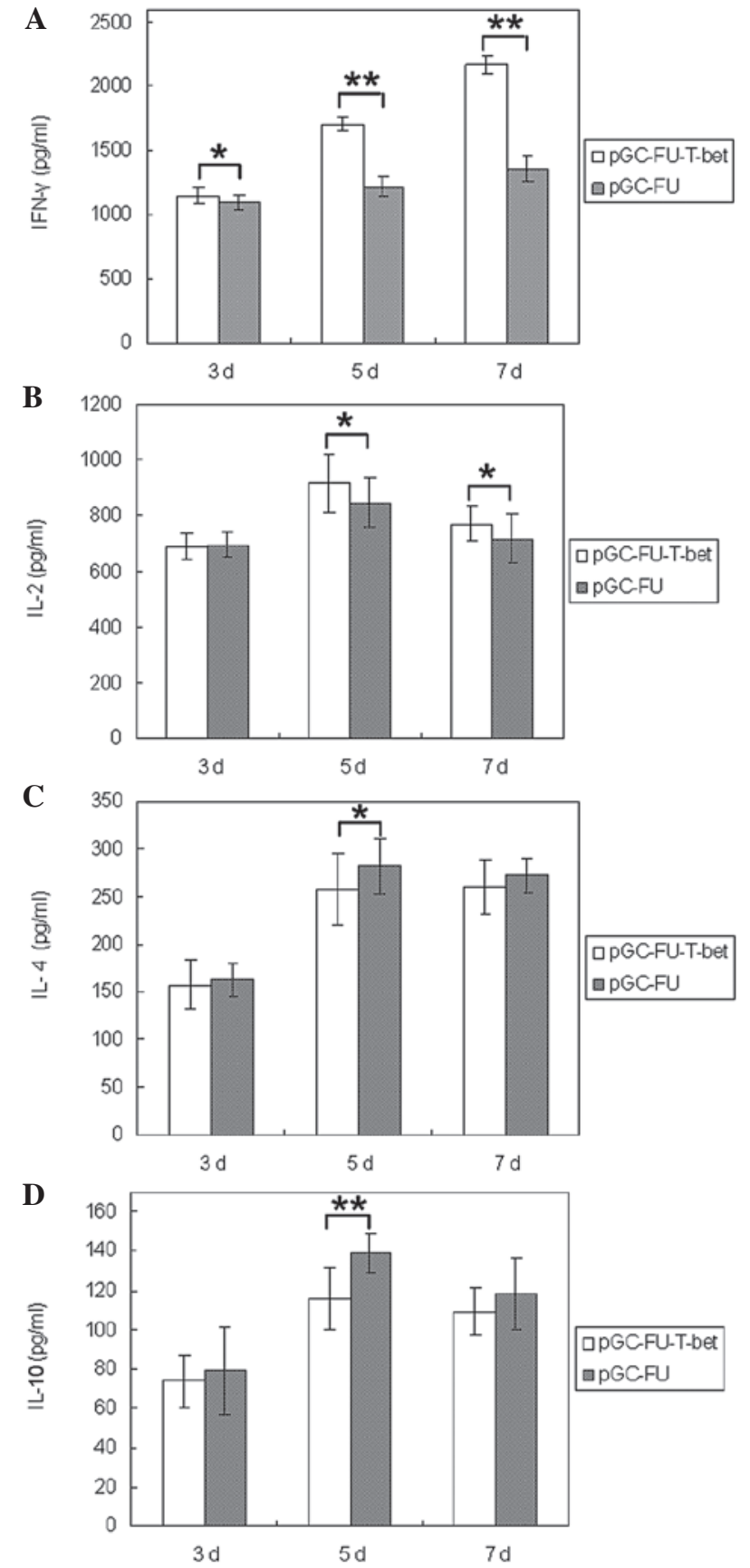

Figure 2. Cytokine assay of $\mathrm{CD}^{+} \mathrm{T}$ cells from peripheral blood of chronic hepatitis $\mathrm{B}(\mathrm{CHB})$ patients $(\mathrm{n}=22)$ following transfection with the T-bet-expressing lentiviral vector (pGC-FU-T-bet) and the no-load lentiviral vector (pGC-FU). The levels of (A) IFN- $\gamma$, (B) IL-2, (C) IL-4 and (D) IL-10 in cultured supernatants were determined on days 3,5 and 7 using enzyme-like immunosorbent assays (ELISAs) $\left({ }^{* *} \mathrm{P}<0.01,{ }^{*} \mathrm{P}<0.05\right)$. T-bet, T-box expressed in $\mathrm{T}$ cells.

T cell priming early in the infection and subsequent development of a quantitatively and qualitatively ineffective CD8 cytotoxic T-lymphocyte (CTL) response. The HBV-specific CTL response plays a fundamental role in viral clearance and pathogenesis of liver disease. The peripheral blood CTL response in chronically infected patients is weak and narrowly focused (7). During HBV infection a balance between the virus and the host's immune system is established, and, apart from CTL, the Th1-type cytokine IFN- $\gamma$, may also exert a 
A

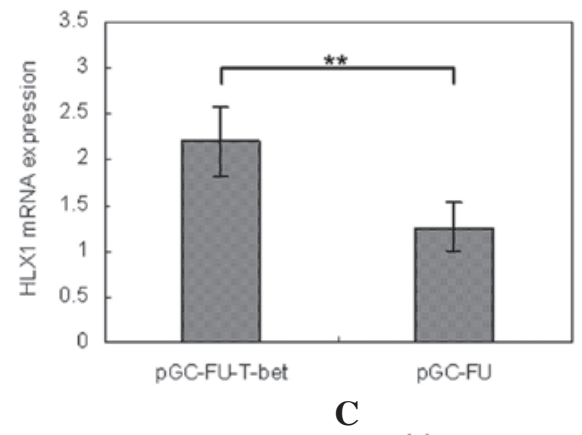

B

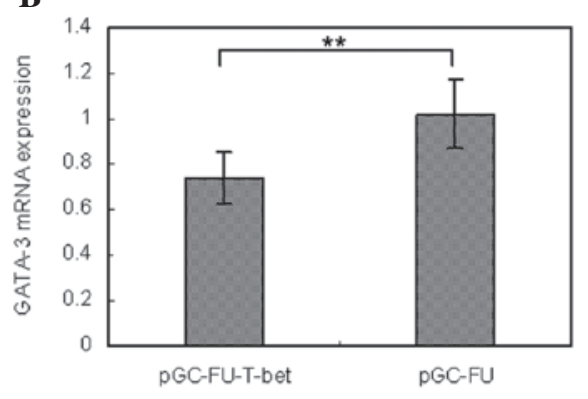

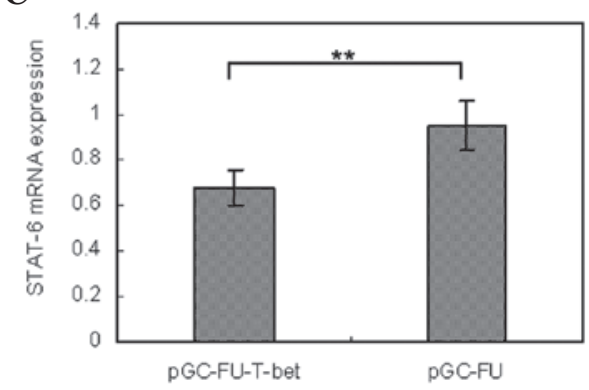

Figure 3. Expression of (A) HLX1, (B) GATA-3 and (C) STAT-6 mRNA was examined at day 5 after infection with the T-bet-expressing lentiviral vector (pGC-FU-T-bet) and the no-load lentviral vector (pGC-FU) $\left({ }^{* *} \mathrm{P}<0.01\right)$. HLX1, H2.0-like homeobox; T-bet, T-box expressed in T cells.

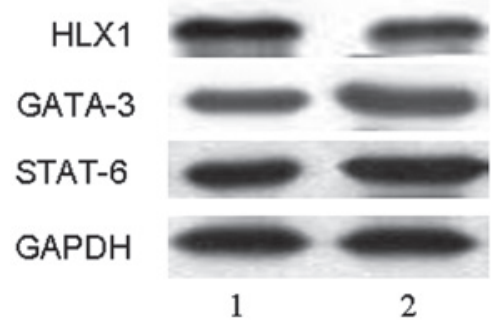

Figure 4. Expression of HLX1, GATA-3 and STAT-6 were examined at day 5 after infection with the T-bet-expressing lentiviral vector. 1, T-bet-expressing lentiviral vector (pGC-FU-T-bet); 2, no-load lentviral vector (pGC-FU). HLX1, H2.0-like homeobox; T-bet, T-box expressed in T cells.

control on HBV replication $(8,9)$. Th1 cells may predominantly contribute to recovery from disease by promoting a cell immune response, which is mediated by producing protective antibodies and enhancing the activity of natural killer (NK) and macrophage cells, particularly CTL in CHB patients $(10,11)$. Host immune reactions biased towards Th2 with production of IL-4 in HBV-infected individuals have been attributed to persistent HBV infection (12). Finally, an imbalance between Th1/Th2 responses may be responsible for the dysregulated immune status in the progression of HBV infection. However, the molecular mechanism involved in this process remains unclear.

T-bet is the master regulator of Th1 development. A link between T-bet expression and immune responsiveness causing HBV infection has been demonstrated by the identification that T-bet expression in CHB patients was decreased when compared with that of AHB patients. In accordance with this finding, it has been reported that T-bet was downregulated during chronic LCMV compared to expression in acute LCMV infection (13). This indicated that deficiency of T-bet during chronic viral infection may contribute to the stimulative effect of T-bet on protective responses in chronic HBV infection.

Lentiviral introduction of T-bet into peripheral blood $\mathrm{CD}^{+} \mathrm{T}$ cells is a likely candidate for regulating the Th1/Th2 immune response. Lentiviral vectors are uniquely suited for gene delivery applications due to their capacity for integrating large transgenes into genomes, maintaining persistent gene expression, efficient transduction of dividing and non-dividing cells, low genotoxicity due to insertional mutagenesis, and weak anti-vector host immunity (14-17). To further study the function of T-bet overexpression in CHB patients, cytokine production was assessed after $\mathrm{CD} 4^{+} \mathrm{T}$ cells were transfected with T-bet-expressing lentivirus. When T-bet was overexpressed, there was increased expression of Th1-type cytokines, IFN- $\gamma$ and IL-2, and decreased expression of Th2-type cytokines, IL-4 and IL-10. These results established that overexpression of T-bet stimulated a Th1-dependent immune response in $\mathrm{CHB}$ patients. $\mathrm{T}$ cell proliferation was found to be indirectly promoted by activation of IL-2 transcription (18), and Th1 differentiation required partial revision to include the major, recently observed, contribution of IL-2 (19). Further studies are required to identify whether IL-2 contributed to a slight Th1 skewing induced by overexpression of T-bet.

In addressing the mechanism by which the polarization of Th cells towards Th1 type occurs when T-bet is overexpressed, the results highlighted the significance of HLX1, given the elevation of HLX1 in $\mathrm{CD}^{+}{ }^{+} \mathrm{T}$ cells transfected with T-bet-expressing lentivirus. HLX1 was specifically expressed in Th1 cells at high levels. Kinetic studies have demonstrated that HLX1 was expressed at a steady level in naïve $\mathrm{CD}^{+} \mathrm{T}$ cells, and upregulated upon commitment to Th1 lineage (20). Mullen et al demonstrated that HLX1 induced by interacting with T-bet and enhanced T-bet-mediated IFN- $\gamma$ production (21). HLX1 also induced IFN- $\gamma$ expression when 
overexpressed at an early time matching the natural time course of HLX1 upregulation during Th1 cell differentiation. When expressed as a transgene in $\mathrm{CD}^{+}{ }^{+} \mathrm{T}$ cells, HLX1 prevented the silencing of the IFN- $\gamma$ gene in $\mathrm{CD}^{+} \mathrm{T}$ cells undergoing Th2 cell differentiation, which resulted in additional cells producing IFN- $\gamma$ (22). However, it remains unclear whether HLX1 is involved in T-bet-induced bias towards developing a Th1 cytokine response in patients with CHB. This study elucidated these observations by examining the expression of HLX1. The results demonstrated that HLX1 was elevated in $\mathrm{CD}^{+} \mathrm{T}$ cells with increasing Th1 cytokine production after lentiviral introduction of T-bet. Evidence supporting the involvement of HLX1 in Th1 cytokine augmentation induced by lentiviral expression of T-bet has been observed, suggesting a HLX1-dependent contribution to the shift of Th cells in favor of Th1 induced by T-bet in patients with CHB.

GATA-3 is considered a 'master regulator' of Th2 development based on its ability to promote Th2 commitment when ectopically expressed in cells under various regulatory situations $(23,24)$. Inhibition of GATA-3 using either small interfering RNA or conditional deletion significantly limited Th2 differentiation and cytokines $(25,26)$. T-bet inhibited GATA-3 binding to target genes, thus suppressing Th2 differentiation (27). Based on these observations, it is plausible to consider that GATA-3 is involved in the inhibition of Th2 cytokines induced by T-bet overexpression in patients with CHB. In this study, a modest reduction in GATA-3 transcription and $\mathrm{mRNA}$ in $\mathrm{CD}^{+}{ }^{+} \mathrm{T}$ cells infected with T-bet-expressing lentivirus was observed, suggesting that repressed GATA-3 contributed to T-bet-mediated limited differentiation into a Th2 lineage in patients with CHB. Th2 cells are also generated by activating naïve $\mathrm{T}$ cells through TCR crosslinking in the presence of exogenous IL-4 whose receptor signals are transduced by STAT-6. STAT-6 which is involved in upstream signaling of GATA-3, has physiological importance for the Th2 program, which can be largely attributed to its ability to synergize with TCR to upregulate GATA-3 (28). The expression of Th2 cytokines, including IL-4, IL-5 and IL-13, was diminished in STAT- $6^{-/-}$mice (29). The regulatory effect of STAT-6 on GATA-3 function and Th2 cytokine production raised the possibility that $\mathrm{Th} 2$ cytokine suppression induced by lentivirally overexpressed T-bet was partly mediated by STAT-6. CD4 ${ }^{+} \mathrm{T}$ cells infected with T-bet-expressing lentivirus demonstrated significantly reduced STAT-6 transcript and mRNA levels, suggesting that T-bet overexpression destabilized Th2 cell development by interfering with STAT-6 expression. This observation established a link between T-bet overexpression and STAT-6, and subsequently Th2 cell development inhibition. However, it remains uncertain whether this STAT-6 effect was or was not GATA-3-independent. Detailed future studies are required to experimentally address this possibility.

Based on the present findings, it is possible to conclude that T-bet was suppressively expressed in CHB patients. Lentivirally overexpressed T-bet induced regulation of Th1/ Th2 immune responses in CHB patients. T-bet overexpression may be immunostimulatory for Th1 type responses mediated by HLX1, which have important implications for enhancing weak immunity in CHB patients. In contrast, lentivirally overexpressed T-bet suppressed the development of Th2 type responses via interacting with GATA-3 and STAT-6. T-bet-expressing lentivirus warrants further investigation as a possible target and as a candidate therapeutic measure for stimulating the immune response and establishing control of infection in $\mathrm{CHB}$ patients.

\section{References}

1. Guidotti LG and Chisari FV: Immunobiology and pathogenesis of viral hepatitis. Annu Rev Pathol 1: 23-61,2006.

2. Asabe S, Wieland SF, Chattopadhyay PK, et al: The size of the viral inoculum contributes to the outcome of hepatitis B virus infection. J Virol 83: 9652-9662, 2009.

3. Lewis MD, Miller SA, Miazgowicz MM, Beima KM and Weinmann AS: T-bet's ability to regulate individual target genes requires the conserved T-box domain to recruit histone methyltransferase activity and a separate family member-specific transactivation domain. Mol Cell Biol 27: 8510-8521, 2007.

4. Beima KM, Miazgowicz MM, Lewis MD, Yan PS, Huang TH and Weinmann AS: T-bet binding to newly identified target gene promoters is cell type-independent but results in variable context-dependent functional effects. J Biol Chem 281: 11992-12000, 2006

5. Pai SY, Truitt ML and Ho IC: GATA-3 deficiency abrogates the development and maintenance of T helper type 2 cells. Proc Natl Acad Sci USA 101: 1993-1998, 2004.

6. Ansel KM, Djuretic I, Tanasa B and Rao A: Regulation of Th2 differentiation and I14 locus accessibility. Annu Rev Immunol 24: 607-656, 2006

7. Gutiérrez-García ML,Fernandez-Rodriguez CM,Lledo-NavarroJL and Buhigas-Garcia I: Prevalence of occult hepatitis B virus infection. World J Gastroenterol 17: 1538-1542, 2011.

8. Raimondo G, Pollicino T, Cacciola I and Squadrito G: Occult hepatitis B virus infection. J Hepatol 46: 160-170, 2007.

9. Mizukoshi E, Sidney J, Livingston B, et al: Cellular immune responses to the hepatitis B virus polymerase. J Immunol 173: 5863-5871, 2004.

10. Brooks DG, Teyton L, Oldstone MB and McGavern DB: Intrinsic functional dysregulation of CD4 T cells occurs rapidly following persistent viral infection. J Virol 79: 10514-10527, 2005.

11. Jiang R, Feng X, Guo Y, et al: $\mathrm{T}$ helper cells in patients with chronic hepatitis B virus infection. Chin Med J (Engl) 115: 422-424, 2002.

12. Akpolat N, Yahsi S, Godekmerdan A, Demirbag K and Yalniz M: Relationship between serum cytokine levels and histopathological changes of liver in patients with hepatitis B. World J Gastroenterol 11: 3260-3263, 2005.

13. Kao C, Oestreich KJ, Paley MA, et al: Transcription factor T-bet represses expression of the inhibitory receptor PD-1 and sustains virus-specific $\mathrm{CD}^{+} \mathrm{T}$ cell responses during chronic infection. Nat Immunol 12: 663-671, 2011.

14. Kootstra NA and Verma IM: Gene therapy with viral vectors. World J Gastroenterol 43: 413-439, 2003.

15. Montini E, Cesana D, Schmidt M, et al: The genotoxic potential of retroviral vectors is strongly modulated by vector design and integration site selection in a mouse model of HSC gene therapy. Clin Invest 119: 964-975, 2009.

16. Abordo-Adesida E, Follenzi A, Barcia C, et al: Stability of lentiviral vector-mediated transgene expression in the brain in the presence of systemic antivector immune responses. Hum Gene Ther 16: 741-751, 2005.

17. Mátrai J, Chuah MK and VandenDriessche T: Recent advances in lentiviral vector development and applications. Mol Ther 18: 477-490, 2010.

18. Meuer SC, Hussey RE, Cantrell DA, et al: Triggering of the T3-Ti antigen-receptor complex results in clonal T-cell proliferation through an interleukin 2-dependent autocrine pathway. Proc Natl Acad Sci USA 81: 1509-1513, 1984.

19. Liao W, Lin JX, Wang L, Li P and Leonard WJ: Modulation of cytokine receptors by IL-2 broadly regulates differentiation into helper T cell lineages. Nat Immunol 12: 551-559, 2011.

20. Zheng WP, Zhao Q, Zhao X, et al: Up-regulation of Hlx in immature Th cells induces IFN-gamma expression. J Immunol 172: 114-122, 2004.

21. Mullen AC, Hutchins AS, High FA, et al: Hlx is induced by and genetically interacts with T-bet to promote heritable $\mathrm{T}(\mathrm{H}) 1$ gene induction. Nat Immunol 3: 652-658, 2002. 
22. Hamalainen-Laanaya HK, Kobie JJ, Chang C and Zeng WP Temporal and spatial changes of histone $3 \mathrm{~K} 4$ dimethylation at the IFN-gamma gene during Th1 and Th2 cell differentiation. J Immunol 179: 6410-6415, 2007.

23. Farrar JD, Ouyang W, Löhning M, et al: An instructive component in T helper cell type 2 (Th2) development mediated by GATA-3. J Exp Med 193: 643-650, 2001.

24. Nawijn MC, Dingjan GM, Ferreira R, et al: Enforced expression of GATA-3 in transgenic mice inhibits Th1 differentiation and induces the formation of a T1/ST2-expressing Th2-committed T cell compartment in vivo. J Immunol 167: 724-732, 2001.

25. Skapenko A, Leipe J, Niesner U, et al: GATA-3 in human T cell helper type 2 development. J Exp Med 199: 423-428, 2004
26. Zhu J, Min B, Hu-Li J, et al: Conditional deletion of Gata3 shows its essential function in $\mathrm{T}(\mathrm{H}) 1-\mathrm{T}(\mathrm{H}) 2$ responses. Nat Immunol 5: 1157-1165, 2004.

27. Hwang ES, Hong JH and Glimcher LH: IL-2 production in developing Th1 cells is regulated by heterodimerization of RelA and T-bet and requires T-bet serine residue 508. J Exp Med 202: 1289-1300, 2005.

28. Zhu J, Guo L, Watson CJ, Hu-Li J and Paul WE: Stat6 is necessary and sufficient for IL-4's role in Th2 differentiation and cell expansion. J Immunol 166: 7276-7281, 2001.

29. Goenka S and Kaplan MH: Transcriptional regulation by STAT6. Immunol Res 50: 87-96, 2011. 